\title{
ABORDAGEM ODONTOLÓGICA EM PACIENTES IDOSOS PORTADORES DA DOENÇA DE PARKINSON
}

\author{
Dental approach in elderly patients with Parkinson's disease
}

\author{
Ana Paula de Freitas Castro ${ }^{1}$ \\ Camila Santos Pereira ${ }^{2}$ \\ Mychelle Percília Souza Santos ${ }^{3}$ \\ Vivian Alkmim Alves ${ }^{4}$ \\ Maria Cleonice de Oliveira Nobre ${ }^{5}$ \\ Maria de Lourdes Carvalho Bonfim ${ }^{6}$
}

Resumo: A Doença de Parkinson (DP) é um distúrbio crônico, progressivo e degenerativo, geralmente inicia-se na meia-idade ou idade avançada. A DP, ao longo do tempo, dificulta ou impossibilita a realização de algumas atividades diárias. É a segunda doença neurodegenerativa mais comum. Objetivos: o objetivo é revisar a literatura sobre as manifestações bucais da DP e descrever os manejos e cuidados adequados antes, durante e após o atendimento odontológico dos pacientes com

\footnotetext{
${ }^{1}$ Graduanda do curso de Odontologia pela Universidade Estadual de Montes Claros (Unimontes), Campus Universitário Prof. Darcy Ribeiro, Montes Claros - MG, 39401-089, Brasil. $\square$ anapfcastro5@gmail.com. https://orcid.org/0000-0002-8296-8781.

${ }^{2}$ Graduanda do curso de Odontologia pela Universidade Estadual de Montes Claros (Unimontes), Campus Universitário Prof. Darcy Ribeiro, Montes Claros - MG, 39401-089, Brasil. $₫$ camilassantos021@ gmail.com. (1) https://orcid.org/0000-0002-4990-8111.

${ }^{3}$ Graduanda do curso de Odontologia pela Universidade Estadual de Montes Claros (Unimontes), Campus Universitário Prof. Darcy Ribeiro, Montes Claros - MG, 39401-089, Brasil. $₫$ mychellepercilia@ gmal.com. https://orcid.org/0000-0001-9036-4972.

${ }^{4}$ Graduanda do curso de Odontologia pela Universidade Estadual de Montes Claros (Unimontes), Campus Universitário Prof. Darcy Ribeiro, Montes Claros - MG, 39401-089, Brasil. \ vvianalkmimalves@ gmail.com. (1) https://orcid.org/0000-0002-4836-7552.

${ }^{5}$ Especialista em Administração dos Serviços de Saúde (UNAERP) e em Metodologia do Ensino Superior (Unimontes). Professora do curso de Odontologia da Universidade Estadual de Montes Claros (Unimontes), Campus Universitário Prof. Darcy Ribeiro, Montes Claros - MG, 39401-089, Brasil. $\square$ cleonicenobre@yahoo.com.br. https://orcid.org/0000-0001-9139-1278.

${ }^{6}$ Doutora em Odontologia em Saúde Coletiva (UFMG). Professora do curso de Odontologia da Universidade Estadual de Montes Claros (Unimontes), Campus Universitário Prof. Darcy Ribeiro, Montes Claros - MG, 39401-089, Brasil. $₫$ maria.bonfim@unimontes.br. https://orcid.org/0000-0001-6399-3212.
}

$\begin{array}{ccc}\text { Recebido em } & \text { Aceito em } & \text { Publicado em } \\ 05 / 03 / 2021 & 21 / 07 / 2021 & 30 / 09 / 2021\end{array}$


Parkinson. Método:trata-se de uma revisão integrativa de literatura, com base em 33 referências, incluindo estudos originais. Resultados: a DP manifesta-se principalmente por problemas motores, que afetam a realização da higiene oral. Pode-se também, observar manifestações bucais: disfagia, sialorréia, xerostomia, sensação de ardor oral, dificuldade na adaptação de próteses dentárias. Dentre as medidas indicadas na abordagem odontológica está: acessibilidade ao consultório, inclinação correta da cadeira, atendimento após 60 a 90 minutos da tomada do medicamento.Considerações finais: Para um bom atendimento odontológico ao paciente com DP, é necessária a abordagem consciente, considerando as doenças presentes. Sendo assim, torna-se imperativo que o cirurgiãodentista conheça os sinais, sintomas e terapia medicamentosa da DP e implicações bucais para um plano de tratamento correto, condutas adequadas e respeito às limitações do paciente.

Palavras-chave: Doença de Parkinson; Higiene oral; Manifestações bucais; Conduta.

Abstract: Parkinson's Disease (PD) is a chronic, progressive and degenerative disorder that usually starts in middle age or old age. PD, over time, makes it difficult or impossible to perform some daily activities. It is the second most common neurodegenerative disease. Objectives: The objective is to review the literature about the oral manifestations of PD and describe the appropriate management and care before, during and after dental care for patients with Parkinson. Method: this is an integrative literature review, based on 33 references, including original studies. Results: PD is manifested mainly by motor problems, which affect the performance of oral hygiene.It is also possible to observe oral manifestations: dysphagia, sialorrhea, xerostomia, oral burning sensation, difficulty in adapting dental prostheses. Among the measures indicated in the dental approach are: accessibility to the office, correct chair inclination, attendance after 60 to 90 minutes of taking the medication. Conclusion: For a good dental care for patients with PD, a conscious approach is necessary, considering the present diseases. Therefore, it is imperative that dentists know the signs, symptoms and drug therapy of PD and oral implications for a correct treatment plan, appropriate conduct and respect for the patient's limitations.

Keywords: Parkinson's disease; Oral hygiene; Oral manifestations; Conduct.

\section{INTRODUÇÃO}

A doença de Parkinson (DP) é um transtorno neurológico complexo, progressivo de longa duração caracterizado pela degeneração das células da parte compacta da substância negra e do locus ceruleus. Essa doença inclui a identificação de inclusões eosinofílicas intracitoplasmáticas constituídas por estruturas protéicas - corpos de Lewis - em neurônios remanescentes da substância negra. O início das manifestações clínicas se dá quando cerca de $60 \%$ dos neurônios dessa região são perdidos. ${ }^{1},{ }^{2}$

Configura como a segunda alteração neurodegenerativa mais comum, perdendo apenas para a doença de Alzheimer. Sua prevalência excede 1\% em idosos com idade superior a 65 anos, sendo que a frequência é aumentada com a idade. A DP corresponde ao parkinsonismo primário, todavia há outros tipos de parkinsonismo, tais como os secundários 
cujas alterações neurológicas são induzidas por medicamentos, hipóxia por infecções, metabólicos, toxinas, traumatismos ou tumores. Existem ainda as síndromes Parkinsionismoplus e as heredodegenerativas. Todo paciente com doença de Parkinson tem parkinsonismo, mas nem todo parkinsonismo se enquadra na doença de Parkinson, mesmo que os sinais cardinais das alterações sejam as mesmas..$^{12,3}$

Os sinais cardinais que determinam esta doença incluem a bradicinesia, rigidez, tremor de repouso e instabilidade postural. De acordo com a estrutura corpórea afetada, percebem-se sintomas e sinais parkinsonianos relacionados, como fácies inexpressivas ou hiponímia, micrografia, fala hipofônica, perda de mobilidade de membro superior na marcha, excesso de saliva na boca, limitação para executar atividades de vida diária (AVD) ou, ainda, aumento do tempo para fazer as refeições. ${ }^{1}$

Os déficits motor e cognitivo, presentes na DP, traduz em comprometimento na higienização desses pacientes. Isto interfere na frequência e na qualidade da higiene oral, elevando a prevalência da falta desta atividade nos pacientes com DP. Na literatura, algumas afecções bucais estão associadas a DP, como a disfagia, sialorréia, xerostomia, sensação de ardor oral, a cárie dentária e a doença periodontal. Nesse contexto, o cirurgião-dentista possui um papel muito importante para minimizar as limitações que o paciente com doença de Parkinson pode ter, tratando-o com muita atenção, paciência e perseverança.4 Dessa maneira, com o intuito de facilitar a condução dos atendimentos clínicos, o objetivo deste estudo é revisar a literatura sobre manifestações bucais da DP, e descrever os manejos e cuidados adequados antes, durante e após o atendimento odontológico dos pacientes idosos com Parkinson.

\section{MÉTODOS}

\section{Primeira etapa}

Recorreu-se a uma revisão narrativa de literatura para o desenvolvimento da presente proposta. Foram utilizadas as ferramentas de buscas gerais e bancos de dados especializados, principalmente o PubMed, Medline Plus, Science Direct, Scielo e Google Acadêmico. Para encontrar tópicos relevantes,utilizou-se de palavras-chave obtidas no guia $\mathrm{MeSH}$, como: "Parkinson's disease", "Parkinson's dentistry", "Oral health" e "Dental 
management", e descritores em português obtidos no DeCS da BVS, como "Doença de Parkinson" e " Odontogeriatria".

\section{Segunda etapa}

Artigos em língua inglesa e portuguesa, incluindo revisões de literatura, trabalhos originais (estudos de coorte prospectivos ou retrospectivos), relato de casos e caso-controle, foram selecionados, totalizando 71 artigos, a partir do ano de 1997. Livros texto foram utilizados como base introdutória para descrever a Doença de Parkinson, tais como: Tratado de Medicina Interna de Wyngaarden, Smith e Bennett (1993); Conhecendo Melhor a Doença de Parkinson de Limongi (2001); Fisioterapia: avaliação e tratamento de O'Sullivan e Schmitz (2004); Doença de Parkinson: aspectos neuropsicológicos de Rocha (2004); Distúrbios neurológicos adquiridos: fala e deglutição de Ortiz (2006); Handbook of Neurochemistry and Molecular Neurobiology: degenerative diseases of the nervous system de Berg e colaboradores (2007); Neurologia Clínica de Harrison de Hauser e Josephson (2009); Tratado de Neurologia de Rowland e Pedley (2011); Odontogeriatria: uma visão gerontológica de Brunetti-Montenegro e Marchini (2013); Tratado de Geriatria e Gerontologia de Freitas e colaboradores (2013); Doença de Parkinson: Estratégias atuais de tratamento de Andrade e colaboradores (2014) e O paciente com necessidades especiais na odontologia de Varellis (2017). Todos os estudos foram avaliados pela leitura inicial de resumos e posteriormente de conteúdos na íntegra, com o objetivo de entender os assuntos relacionados a DP e assim avaliar as condutas adequadas no tratamento odontológico do paciente portador da Doença de Parkinson. Utilizou-se, ao final, 33 referências para a confecção do presente artigo.

\section{RESULTADOS}

As manifestações clínicas da DP surgem a partir de cada área acometida e acusa distúrbios motores e não motores. Os distúrbios motores como a tétrade clássica (tremor em repouso, rigidez, bradicinesia e instabilidade postural)aparece quando há depleção de dopamina na parte compacta da substância negra. Para haver repercussão clínica, a perda desses neurônios é superior a $60 \%$.

Nos sintomas não motores, o que contribui para o seu aparecimento é a diminuição da dopamina, as catecolaminas e serotonina. Pode-se incluir as disfunções 
autonômicas (hipotensão, constipação), anosmia, parestesia, ansiedade, depressão, distúrbios do sono (acometimento de substância reticular) e, com a evolução da doença, o consequente acometimento do neocórtex leva a distúrbios cognitivos e demência. ${ }^{6}$ Além disso, pode-se citar a diminuição do olfato, que é um dos primeiros sintomas a ser considerados por alguns autores. Raramente, é complexa bilateral, independente da terapia com dopamina. ${ }^{7}$ Está presente em cerca de $90 \%$ dos pacientes com parkinson. ${ }^{8}$

Ainda sobre sintomas não motores, as manifestações bucais da DP, enfoque deste trabalho, incluem disfagia, sialorréia, xerostomia, sensação de ardor oral, alteração da saúde oral (cárie e doença periodontal) e uso e a adaptação de próteses dentárias. ${ }^{9}$ Em relação à disfagia, esta ocorre devido a movimentos da musculatura oral lentos e dificuldades na coordenação das ações bucais. É caracterizada pelo engasgamento ao engolir provocado por alteração na deglutição, e ocorre principalmente ao consumir líquidos. ${ }^{10}$ No atendimento odontológico, tal manifestação associada ao comprometimento do reflexo de vômito aumentam o risco de aspiração de fluidos orais e de irrigação, por isso é importante remover os fluidos da cavidade oral rapidamente, a fim de reduzir o risco de pneumonia por aspiração. ${ }^{11}$

Uma manifestação oral comum na disfagia é o refluxo ácido, que pode causar erosão dentária e consequentemente hipersensibilidade dentinária. Por isso, é recomendado que o paciente (ou cuidador responsável) espere cerca de 30 minutos após um episódio de refluxo ácido para realizar a escovação dentária e assim minimizar os danos ao esmalte dentário. $^{12}$

A disfagia pode levar também a outro distúrbio comum em pacientes com DP, a sialorréia, que ocorre devido às dificuldades de mastigação e deglutição, além da hipotonicidade na musculatura facial. ${ }^{9}$ Ocorre em $32-74 \%$ dos pacientes e sua principal característica é o aumento e acúmulo da saliva na cavidade oral, o que pode causar um impacto significativo na qualidade de vida desses pacientes. ${ }^{13,14}$

Quando o paciente apresenta sialorréia, uma das ocorrências relatadas é a dificuldade de manutenção de um campo operatório seco em alguns procedimentosodontológicos. Sendo assim, o tratamento odontológico em pacientes com DP deve ser realizado em uma posição relativamente ereta, para minimizar as dificuldades 
durante o atendimento, principalmente em procedimentos complexos no arco maxilar ou na cavidade oral posterior. ${ }^{11}$

A xerostomia, sensação de boca seca, afeta cerca de 55\% dos pacientes com DP. ${ }^{14}$ Ela está associada ao uso dos medicamentos utilizados no tratamento da DP, que incluem fármacos dopaminomiméticos, anticolinérgicos e amantadina. A xerostomia também está relacionada ao aparecimento de alterações periodontais e cáries, uma vez que funções importantes da saliva como ação antibacteriana, remineralização e lubrificação não estão mais presentes. ${ }^{12,14,15}$

Com o propósito de aliviar a xerostomia, pode-se recomendar várias estratégias, tais como: beber água com frequência, utilizar goma de mascar sem açúcar ou lançar mão de terapia farmacológica. $\mathrm{O}$ uso de saliva artificial pode ser muito útil em alguns casos, principalmente o uso de substitutos salivares contendo fluoretos, que reduzem o risco de cárie. ${ }^{12}$ Com a redução no fluxo salivar o paciente também pode apresentar queilite angular, mucosites, infecções fúngicas por cândida, além de estar suscetível ao surgimento de cárie dentária e doença periodontal. ${ }^{15}$

Outra manifestação em pacientes com Parkinson é a síndrome da ardência bucal. Este distúrbio é caracterizado por uma sensação de dor e queimação intraoral, não possuindo correspondência física ou laboratorial. ${ }^{14}$ Normalmente, está associada a medicação com levodopa, que promove a mastigação parafuncional e pode derivar de deficiências minerais, vitaminas ou desequilíbrios hormonais. ${ }^{12}$

Os locais normalmente afetados pelo ardor bucal são a língua, lábios, rebordo alveolar e palato duro. ${ }^{9}$ A presença da síndrome da ardência bucal também pode ser influenciada por fatores psicológicos como ansiedade, depressão, estresse e distúrbios compulsivos. ${ }^{14} \mathrm{O}$ tratamento varia, podendo ser empregada terapia antifúngica, uso do clonazepam, terapia cognitiva-comportamental, reposição de vitaminas ou utilização de ácido alfa-lipóico. ${ }^{9}$

Os pacientes com DP têm tendência para ter uma saúde oral e periodontal comprometida devido à falta de controle muscular orofacial, hipo ou hipersalivação. ${ }^{16,17} \mathrm{~A}$ dificuldade de higiene oral no paciente com DP também é influenciada pelas limitações motoras presentes neste paciente, promovendo um grande acúmulo de biofilme e a instalação da cárie dentária e doença periodontal. ${ }^{10}$ Indivíduos com DP apresentam mais 
envolvimento periodontal, incluindo recessão gengival, mobilidade e perda dentária, do que indivíduos sem a doença. A cárie radicular também pode ser uma preocupação, uma vez que as exposições das superfícies radiculares aumentam com o envelhecimento e a população com DP tem, em média, mais de 50 anos. ${ }^{12}$

Muitos pacientes com Parkinson tendem a preferir alimentos açucarados devido a alterações no paladar, que ocorrem com o decorrer da idade e com o surgimento de doenças degenerativas. No entanto, o consumo de alimentos ricos em açúcar também pode aumentar o acúmulo de placa, bem como predispor a doença periodontal e cárie dentária. ${ }^{9}$ Os distúrbios cognitivos e motores causados pela DP podem piorar a progressão da Doença Periodontal. $^{18}$

O controle e a retenção da prótese em boca dos pacientes com DP estão comprometidos. Isto ocorre devido à espessura viscosa de saliva, xerostomia, alteração da tonicidade muscular, perda de peso e alterações estruturais da boca. O componente psicológico, como depressão, problemas cognitivos e apatia, também pode comprometer o sucesso do uso da prótese. 3,17

A prevenção é fundamental para a manutenção da saúde oral, sendo bastante importante que o paciente seja acompanhado pelo dentista quando é diagnosticado com DP. Deve ser realizada uma história clínica completa e um exame clínico abrangente. ${ }^{12} \mathrm{Com}$ o avançar da doença, o aumento na complexidade dos sintomas apresentados exige que o paciente receba atenção multidisciplinar. ${ }^{15}$ Esta atenção requer uma equipe, a qual inclui médico, cirurgião dentista, fisioterapeuta, fonoaudiólogo, psicólogo, nutricionista, bem como familiares e cuidadores formais. ${ }^{4,12}$

\section{Abordagem Odontológica:}

Os cuidados no atendimento odontológico ao paciente com DP envolvem uma conduta com adequada atenção, paciência e perseverança e deve buscar todas as formas possíveis de minimizar as limitações que ele apresenta. ${ }^{19}$ As interações interprofissionais também tornam-se imprescindíveis para atuar diante das necessidade do paciente com Parkinson. ${ }^{10}$

O consultório odontológico deve apresentar acessibilidade, considerando todas as limitações que um paciente com Parkinson, em estágio mais avançado, pode apresentar. ${ }^{10} \mathrm{O}$ local deve possuir rampas de acesso (o que facilita a passagem de cadeiras de 
rodas), ser bem iluminado, o piso deve ser de preferência antiderrapante e a remoção de tapetes ou objetos no local de passagem também é indicada. ${ }^{15}$

A abordagem do paciente com Parkinson deve ser compassiva e atenciosa, afim de incentivar adesão ao tratamento e reduzir a sua ansiedade. O tempo de atendimento também deve ser conduzido de forma eficaz, para favorecer consultas mais rápidas. A comunicação é, igualmente, importante e para facilitar este processo, deve-se fazer perguntas fechadas e esperar que o paciente responda em seu ritmo. ${ }^{17}$ É fundamental que o cirurgião dentista construa uma boa relação com o paciente, conversando com ele, segurando-o de maneira cuidadosa e colocando-o na cadeira em uma posição confortável, a fim de promover uma sensação de segurança. ${ }^{20}$

A presença de um acompanhante é aconselhável, mesmo quando o paciente possua parte de sua autonomia, uma vez que o acompanhante pode complementar informações que foram esquecidas na anamnese e, caso houver necessidade, dar consentimento para a realização de tratamentos,além de fazer com que o paciente se sinta mais confortável. ${ }^{15}$ É importante salientar também que o paciente com DP, quando apresenta demência ou potencial de confusão, não pode ser deixado sozinho em nenhum momento da consulta. ${ }^{12}$

A anamnese deve ser realizada com atenção, já que o plano de tratamento deve ser feito considerando as limitações que o paciente apresenta. ${ }^{20}$ Uma anamnese bem detalhada precisa abranger as limitações físicas e psicológicas apresentadas, a história médica pregressa, medicação utilizada, atividades que o indivíduo é capaz de realizar e se tem alguém responsável por auxiliar nos cuidados desse paciente. ${ }^{4}$ Exames físicos e comportamentais também devem ser avaliados e a pressão arterial do paciente deve ser monitorada. ${ }^{10}$

O atendimento odontológico é mais indicado no período da manhã, no qual os pacientes estão mais descansados e os tremores estão reduzidos. Além disso, o atendimento deve ser feito após 60 a 90 minutos da tomada do medicamento, quando atinge o pico de ação farmacológica. ${ }^{17,20}$ É importante ressaltar também que a duração das consultas não devem ultrapassar mais de 45 minutos por sessão, visto que os pacientes podem não tolerar grandes quantidades de tempo na cadeira. ${ }^{12,15}$ 
A cadeira odontológica não deve ser inclinada mais que $45^{\circ}$, para evitar engasgamento ou asfixia por aspiração. ${ }^{10}$ Outra recomendação importante é levantar a cadeira devagar após o término da consulta, para evitar que o paciente manifeste hipotensão ortostática.17 A hipersudorese e a incontinência também podem ser um problema, por isso, o paciente deve ser orientado a esvaziar a bexiga antes da consulta e o consultório deve ser ajustado a temperatura ambiente. ${ }^{15}$

Procedimentos preventivos, como profilaxia e aplicação de flúor são importantes para evitar o aparecimento de lesões cariosas. ${ }^{4}$ As próteses removíveis poderão ser confeccionadas e instaladas para restabelecer a função. Porém, o paciente precisa ter destreza para mantê-la na cavidade oral. ${ }^{15}$ Quando for possível, uma alternativa viável para reduzir o tempo de trabalho e fazer com que próteses já existentes fiquem mais funcionais é através do reembasamento. ${ }^{19}$

A exodontia só deve ser realizada quando não houver outra alternativa mais conservadora. Também não é indicada a realização de cirurgia periodontal, pois o controle de placa é dificultado; o ideal é que sejam realizados raspagem e alisamento radicular em sessões curtas. ${ }^{21}$ Nos casos em que existam poucas bolsas com profundidade maior que $5 \mathrm{~mm}$, a antibioticoterapia pode ser administrada em conjunto com procedimentos básicos. ${ }^{15}$

O isolamento absoluto pode ser realizado, porém é necessário ficar atento quanto ao acúmulo de saliva abaixo do lençol de borracha, que deve ser sugada rapidamente para evitar sufocamento. Por isso, é recomendada a utilização de sugador de alta potência durante o atendimento. ${ }^{19}$ Ainda assim, desde que seja realizado o correto monitoramento da saliva, o uso do isolamento absoluto pode contribuir protegendo a língua de lesões e a orofaringe da queda de objetos. O trabalho a quatro mãos é indispensável e, dependendo do paciente, pode ser necessário até mesmo o trabalho a 6 mãos. ${ }^{15}$

A anestesia local deve ser administrada com cautela, pois a perda da sensação intensifica a manifestação da disfagia. ${ }^{12} \mathrm{O}$ uso de anestésicos contendo vasoconstritor também não é contra-indicado, mas não deve passar de dois tubetes na dose de 1:100.000, já que o uso de vasoconstritor em pacientes que utilizam levodopa ou entacapona pode elevar a pressão arterial, causar taquicardia e arritmia cardíaca. ${ }^{15}$

Apoiar a cabeça do paciente durante o atendimento pode ajudar a aliviar movimentos involuntários e criar um ambiente de trabalho mais seguro para o paciente e 
para o cirurgião dentista. Em alguns casos, o uso de dispositivos de estabilização podem ser necessários, como nos movimentos involuntários descontrolados, queprovocam risco à segurança do paciente e/ou do clínico, durante o tratamento. ${ }^{12}$ Dentre os dispositivos de contenção estão as correias acolchoadas e fitas de amarração. ${ }^{4}$

Quando a doença atinge estágios avançados, o atendimento odontológico só poderá ser realizado enquanto o paciente estiver sob anestesia geral em ambiente hospitalar. Essa decisão pode ser discutida com os profissionais de saúde envolvidos no cuidado do paciente, para garantir que todos, incluindo familiares e cuidadores, compreendam que esta é a melhor opção de tratamento para o indivíduo com a DP avançada. ${ }^{12,19}$

Os pacientes com DP podem apresentar também dificuldades de realizar a higiene bucal devido a diversos fatores que incluem comprometimento motor, apatia, depressão e demência. Muitas vezes, a progressão da doença leva o cuidador ou um membro da família a assumir a responsabilidade pela higiene bucal, porque a DP avançada impede que o paciente realize o autocuidado oral adequado. ${ }^{14}$ No entanto, enquanto for possível, o paciente deve ter autonomia para realizar sua escovação, podendo lançar mão do uso de escovas elétricas ou adaptadas, como através do uso de uma bola de tênis no cabo. ${ }^{15}$

Tanto os pacientes quanto os cuidadores devem ser instruídos sobre as técnicas de higiene bucal, uso do fio dental e higienização de próteses. ${ }^{4}$ É importante ressaltar que a dentadura deve ser removida durante à noite e, para higienização da língua e gengiva, deve ser utilizada uma escova macia. ${ }^{21}$ Após o atendimento odontológico, todas as orientações de higienização devem ser reforçadas e repassadas por escrito. Vale ressaltar que, após o atendimento,a cadeira deve ser levantada cuidadosamente para evitar hipotensão ortostática. $^{17}$

\section{DISCUSSÃO}

É digno de nota que foram observadas alterações do olfato em cerca de $90 \%$ dos pacientes com DP. ${ }^{8}$ Esta alteração é um marcador precoce e sensível da fase pré-clínica da DP podendo preceder os sintomas motores em pelo menos 4 anos. $^{6,7,8}$

Como uma das manifestações orais da DP, ressalta-se a disfagia. Esta alteração na deglutição é muito comum nos pacientes com Parkinson. ${ }^{22}$ Corroborando com estes autores Friedlander e colaboradores (2009), além de concordarem, descreveram que isto 
ocorre devido às deficiências motoras da faringe. ${ }^{23}$ Em outro estudo, observou-se também que a variação da prevalência das alterações na deglutição, está entre $9 \%$ a $77 \% .{ }^{24}$ Diante dessa manifestação tão comum, é importante que o cirurgião-dentista esteja atento aos pacientes que tenham disfagia, principalmente durante o atendimento odontológico, pois esses indivíduos apresentam um maior risco de contrair pneumonia aspirativa, desnutrição e morbidade psicossocial. ${ }^{9}$

Outra manifestação oral presente na DP é a sialorréia. Ela ocorre devido a dificuldades na mastigação e a perda de tônus muscular facial. ${ }^{25}$ Em um estudo realizado por Nogueira (2016), é relatado que a sialorréia pode ser decorrente da disfagia, já que a primeira ocorre devido a alteração na mastigação e deglutição; observou-se também que sua frequência .ocorre em 32-74\% dos pacientes. ${ }^{9}$ Resultado semelhante, com. uma frequência da sialorréia em até $80 \%$ dos pacientes com DP, também foi observado. ${ }^{26}$

A xerostomia também é outra manifestação bucal, comum nos pacientes com DP - Esta sensação de boca seca contribui para o aparecimento da cárie dental e doença periodontal. ${ }^{9}$ Este achado também foi observado em outros estudos. ${ }^{12,}{ }^{14}$ Alguns indivíduos com DP, que apresentam xerostomia, queixam-se de ulcerações na boca, desconforto oral e problemas na retenção da prótese. ${ }^{12}$

A respeito do uso de próteses dentárias, em pacientes com DP, a perda do controle neuromuscular colabora para a perda da estabilidade de próteses totais. ${ }^{27} \mathrm{Em}$ estudos realizados por Clifford e Finnerty (1995) e Ribeiro e colaboradores (2016) observaram que pacientes com DP que fazem uso de próteses totais sentem desconforto com as mesmas e têm dificuldade na mastigação. ${ }^{27,}{ }^{28}$ É bom lembrar que a função mastigatória prejudicada, junto com problemas relacionados às próteses, podem agravar as dificuldades de mastigação e deglutição já presentes nesses pacientes com DP. ${ }^{29}$

Uma observação importante descrita nos estudos se refere aos dentes cariados. Ao contrário do que se poderia esperar, nos pacientes com DP, o número de dentes cariados é menor. Porém, apresentam um aumento na prevalência de periodontite quando comparado a um grupo controle normal. Autores também descobriram que a frequência de escovação foi maior entre os pacientes com doença de Parkinson, com média de duas escovagens diárias. Os resultados do aumento das taxas de gengivite e periodontite, com aumento significativo da profundidade das bolsas periodontais servem como lembretes de que acúmulos de placas 
podem causar não apenas cáries, mas também perda óssea neste grupo especial de pacientes, devido à sua dificuldade presumida com a higiene bucal diária. ${ }^{3}$

Por outro lado, quando se analisa acometimento apenas pela doença cárie, emboraa higiene bucal seja supostamente ruim em pacientes com Parkinson, o número de lesões cariosas foi tão alto quanto nos grupos controle, sugerindo que tais pacientes não estão protegidos contra a cárie. Além disso, autores também descobriram que os sujeitos estavam cientes da necessidade à boa higiene bucal e relataram escovar os dentes frequentemente. No entanto, isso não foi suficiente. Até mesmo,indivíduos com DP, que usavam escovas de dentes elétricas e fio dental, foram observados resultados insatisfatórios para eficiência no controle de placa, em virtude das limitações motoras que estes pacientes apresentavam. Dessa forma, é imprescindível fortalecer ainda mais a higiene bucal neste grupo, através de uma combinação de métodos físicos e controle químico de placa, com clorexidina ou um agente similar. Ademais, o monitoramento regular deve ser realizado por um dentista ou higienista dentalpara garantir que os pacientes sejam capazes de manter um nível razoável de higiene bucal. ${ }^{3}$

Um estudo de caso-controle realizado em Leiden, na Holanda,comparando o estado de saúde bucal de 74 pacientes com DP e 74 pacientes controle, estas alterações foram demonstradas. Os pacientes com Parkinson apresentavam um estado de saúde bucal enfraquecido e cuidados com a higiene bucal reduzidos quando comparados a um grupo controle ideal de acordo com idade, gênero, origem social e histórico de vida. A maior duração da doença e a maior gravidade foram associadas a mais problemas de saúde bucal e de higiene oral, sugerindo que relacionam-se aos comprometimentos pela doença Parkinson. ${ }^{30}$

Sendo assim, os pacientes com DP poderão apresentar dificuldade na performance da higienização bucal apropriada. Também ficam mais propensos a maus hábitos alimentares, como ingestão de alimentos ricos em açúcar. Ademais as mudanças cognitivas poderão afetar a ida deste paciente até o cirurgião dentista e quando vão; provavelmente alterações comportamentais que dificultam o procedimento odontológico, são possíveis de ocorrer. ${ }^{3}$ Nesse contexto, os cirurgiões-dentistas devem estar habilitados para lidar com os pacientes com DP. Além do mais, a projeção desta doença para o ano 2030 é 
quese duplicará. O número de indivíduos com DP com idade superior a 50 anos, passará de 4,1 e 4,6 milhões em 2005, para 8,7 e 9,3 milhões em 2030. ${ }^{12,31}$

A respeito da importância do trabalho multidisciplinar, as intervenções não farmacológicas, como o trabalho fisioterápico e fonoaudiológico certamente trazem benefícios. ${ }^{1}$ A exemplo disto, pode-se citar a pesquisa transversal quantitativa, realizada na cidade de Curitiba (Paraná) que obteve importante resultados. Foi observado que os pacientes que possuíam quadros mais graves de DP e praticavam as atividades multidisciplinares incluindo a fonoaudiologia, fisioterapia e psicologia obtiveram redução das dosagens de levodopa (resultado estatisticamente significativo) em relação àqueles que não praticavam e estavam no mesmo quadro grave. ${ }^{32}$

Quanto aos procedimentos odontológicos realizados no paciente com DP, cerca de 43,6\% dos profissionais da área da saúde desconhecem a atuação do cirurgião-dentista junto ao portador da doença de Parkinson. No entanto, a atuação da odontologia contribui muito para melhoria da qualidade de vida deste paciente. ${ }^{21}$

A despeito disso, uma abordagem cuidadosa deve ser propostapara o atendimento odontológico destes pacientes com criação de protocolos clínicos. Mas enquanto alguns autores sugerem queas consultas devem ser feitas no melhor horário para o paciente, após 60 a 90 minutos da ingestão do medicamento ${ }^{4}$, outros ainda preconizam a realização do atendimento, pela manhã, com sessões curtas ,além de recomendar também o respeito do período de pico de ação do medicamento antiparkinsoniano no organismo (60 a 90 minutos) para realizar o atendimento odontológico. ${ }^{21}$

Antes do atendimento odontológico, os autores são concordantes em recomendar que os consultórios permitam umaadequada acessibilidade aos pacientes. Aqueles que fazem uso de bengala, andarilho, cadeira de rodas ou outros auxílios devem entrar e se locomover de forma fácil e independente neste ambiente. Outro fator a se considerar é a realização de uma anamnese bem detalhada. Ela deve permitir conhecer toda a história médica, limitações físicas, psicológicas do paciente, bem como a medicação utilizada, qual a melhor hora do dia para os atendimentos odontológicos, quais são asatividades diárias realizadas, e se tem a seu dispor, um cuidador. ${ }^{4,21}$ A presença do cuidador constitui um facilitador pois ele será útil para transmitir confiança ao paciente e interpretar as recomendações dadas pelo clínico. ${ }^{17}$ Após a anamnese e exame clínico, um plano de tratamento individualizado deverá 
ser traçado de acordo com as necessidades do paciente, lembrando sempre de examinar os sinais vitais, pressão arterial, pulso e respiração; devem ser registrados sempre. ${ }^{4}$

Já durante o atendimento, há unanimidade entre os autores em recomendar a não inclinação da cadeira odontológica por mais que $45^{\circ}$. Esta recomendação trará mais conforto para o paciente com DP evitandoproblemas de deglutição apresentados pelo paciente no momento do atendimento. Além disso, deve-se evitar a confecção de coroas e pônticos, uso de isolamento absoluto e extração de elementos dentários, apesar de não serem procedimentos contraindicados. ${ }^{4,21}$

Sendo assim, para Ferreira e colaboradores (2017), a Odontologia preventiva se faz essencial para os pacientes com DP. Ainda, consoante às ideias anteriores, outros autores defendem que o cirurgião dentista deve apostar em procedimentos profiláticos, como profilaxias e aplicação de verniz de flúor, com o intuito evitar o aparecimento de cáries. ${ }^{4,21}$ Em paciente desdentado, deve ser avaliada a qualidade das próteses presentes. É importante também instruir as técnicas de higiene oral, como a técnica de escovação, o uso do fio dental, e limpeza das próteses removíveis. Para pacientes com muitas limitações motoras, pode ser indicado o uso de escova elétrica ou o uso de escova adaptadas. Como medida preventiva, também é necessário que as visitas ao dentista sejam regulares, podendo variar a periodicidade conforme o avanço da doença. Ademais, deve-se apostar na educação para a higiene oral, para evitar a aumento do número de lesões cariosas e controlar a doença periodontal. $^{21,33}$

Nessa perspectiva, a estratégia do plano de tratamento, deve levar em conta desafios físicos e psicológicos associados à própria doença. A gestão desta estratégia deve ser adequada às necessidades do paciente e este deve ser tratado com delicadeza, utilizando uma comunicação eficaz que facilite a compreensão de tudo o que lhe está sendo proposto e orientado. A educação e motivação do paciente e ainda o acompanhamento após o tratamento são fundamentais para o seu sucesso. ${ }^{17}$

\section{CONSIDERAÇÕES FINAIS}

A doença Parkinson é uma doença crônica e progressiva do sistema nervoso central cuja prevalência aumenta com o envelhecimento. As manifestações clínicas da DP surgem a partir de cada área acometida e acusa alterações motoras e não motoras. Dentre as 
características motoras, temos os sinais cardinais da DP,que incluem a bradicinesia, tremor de repouso, rigidez muscular e instabilidade postural. As manifestações não motoras incluem as alterações bucais como disfagia, sialorréia, xerostomia, sensação de ardor oral, doença cárie e doença periodontal.

O tratamento odontológico deve ser, preferencialmente, preventivo e iniciado nos primeiros estágios da doença. Ele deve ser feito de 60 a 90 minutos após a medicação antiparkinsoniana. A cadeira odontológica não deve ter uma inclinação superior a 45 graus. As orientações ou recomendações devem ser passadas para os familiares e cuidadores, a fim de estimular a realização de uma higiene oral de maneira correta e contínua, durante e após o tratamento, para manter uma boa saúde bucal.

Enfim, para que se cumpra efetivamente um bom atendimento odontológico ao paciente com DP, é de extrema importância a abordagem consciente do paciente idoso, levando em consideração as doenças presentes. Sendo assim, torna-se imperativo que o cirurgião-dentista conheça todos os sinais, sintomas e terapia medicamentosada doença de Parkinson e suas implicações bucais para que o plano de tratamento seja traçado da forma correta com condutas clínicas adequadas, sempre respeitando as limitações impostas pelo paciente.

Além disso, é imprescindível que o paciente com Parkinson receba um tratamento interdisciplinar e integral uma vez que as limitações posturais, respiratórias, fonatórias e nutricionais apresentam-se interligadas pelas complexas conexões neuromusculares.

\section{REFERÊNCIAS}

1. FREITAS, Elizabete Viana de et al. Tratado de geriatria e gerontologia. $3^{\mathrm{a}}$ ed. Rio de Janeiro: Guanabara Koogan, 2013.

2. HAUSER, Stephen L.; JOSEPHSON, S. Andrew. Neurologia clínica de Harrison. $3^{\mathrm{a}}$ ed. Porto Alegre: AMGH EDITORA LTDA, 2015.

3. BATISTA, Leonardo M. et al. Oral Hygiene in patients with parkinson'sdisease.Rhode island medical jornal, 2015.

4. MACHADO, Bianca Brito; PIAZERA, Cyrene. Doença de Parkinson e odontologia: uma revisão de literatura narrativa. Revista Ceuma Perspectivas, vol. 30, 2017. 
5. WIRDEFELDT, Karin et al. Epidemiology and etiology of Parkinson's disease: a review of the evidence. European journal of epidemiology, v. 26, n. 1, p. 1, 2011.

6. ROSSO ALZ, Nicaretta DH, Mattos JP. Correlações anatomoclínicas na Doença de Parkinson. Revista Brasileira de Neurologia, v. 44, n.4, 2008.

7. DOTY, Richard L. Olfactory dysfunction in Parkinson disease. Nature Reviews Neurology, v. 8, n. 6, p. 329-339, 2012.

8. HAEHNER, Antje; HUMMEL, Thomas; REICHMANN, Heinz. Olfactory loss in Parkinson's disease. Parkinson's Disease, v. 2011, 2011.

9. NOGUEIRA, A. F. A doença de Parkinson e suas implicações na saúde oral. 2016. Dissertação (Mestrado em Medicina Dentária) - Instituto de Ciências da Saúde Egas Moniz, Portugal, 2016.

10. PISTORI, Juliana; MACHADO, Mariana. A Interprofissionalidade no Tratamento de Pacientes com Parkinson: Uma Análise Odontológica, Bragança Paulista, 2019.

11. NEWADKAR, Ujwala R. et al. Oral health issues and challenges in parkinson's disease. International Journal Of Nutrition, Pharmacology, Neurological Diseases, v. 7, n. 3, p. 54, 2017.

12. DEBOWES, S. L.; TOLLE, S. L.; BRUHN, A. M. Parkinson's disease: considerations for dental hygienists. International journal of dental hygiene, v. 11, n. 1, p. 15-21, 2013.

13. EGEVAD, Gustav; PETKOVA, Valentina Yankova; VILHOLM, Ole Jakob. Sialorrhea in patients with Parkinson's disease: safety and administration of botulinum neurotoxin. Journal of Parkinson's disease, v. 4, n. 3, p. 321-326, 2014.

14. ZLOTNIK, Yairet al. Disorders of the oral cavity in Parkinson's disease and parkinsonian syndromes. Parkinson's disease,. v. 2015, 2015.

15. CARACÓIS, Lénia Matado. Doença de Parkinson no paciente odontogeriátrico. 2012. Dissertação (Mestrado Integrado em Medicina Dentária) - Faculdade de Medicina Dentária, Universidade de Lisboa.

16. BAKKE, Merete et al. Orofacial function and oral health in patients with Parkinson's disease. European journal of oral sciences, v. 119, n. 1, p. 27-32, 2011.

17. HARALUR, Satheesh B. Clinical strategies for complete denture rehabilitation in a patient with Parkinson disease and reduced neuromuscular control. Case reports in dentistry, 2015.

18. KAUR, Tejaswani; UPPOOR, Ashita; NAIK, Dilip. Parkinson's disease and periodontitis-the missing link? A review. Gerodontology, v. 33, n. 4, p. 434-438, 2016.

19. INTROINI, M. B. de P. Protocolo de atendimento odontológico para pacientes com doença de Parkinson. 2009. Monografia (Especialista em Odontogeriatria) - Faculdade de Odontologia de Piracicapa, Universidade Estadual de Campinas, Piracicaba, 2009. 
20. LINS, Carla Cabral dos Santos Accioly; DE ALBUQUERQUE MELO, Antônio Carlos Moura; LIMA, Georgina Agnelo. Atenção à saúde bucal de idosos com Parkinson na Universidade Federal de Pernambuco: Relato de Experiência. RevistaLongeviver, n. 44, 2015.

21. FERREIRA, Beatryz Borges Magalhães et al. A Intervenção Odontológica e a Visão Da Equipe Multidisciplinar em Pacientes Portadores de Parkinson do Hospital Dia Geriátrico de Anápolis. Sci Invest Dent, v. 22, n. 1, p. 76-81, 2017.

22. BAIJENS, Laura WJ et al. Swallowing in Parkinson patients versus healthy controls: reliability of measurements in videofluoroscopy. Gastroenterology research and practice, v. 2011, 2011.

23. FRIEDLANDER, Arthur H. et al. Parkinson Disease: Systemic and Orofacial Manifestations, Medical and Dental Management. J Am Dent Assoc., vol. 140, n. 6, p. 658-69, 2009.

24. BARBE, Anna Greta et al. Self-assessment of oral health, dental health care and oral healthrelated quality of life among Parkinson's disease patients. Gerodontology, v. 34, n. 1, p. 135$143,2017$.

25. EINARSDÓTTIR, Erna Rún et al. Dental health of patients with Parkinson's disease in Iceland. Special Care in Dentistry, v. 29, n. 3, p. 123-127, 2009.

26. SERRANO-DUENAS, M. Sialorrhea in patients with Parkinson. A six year prospective study. Revista de neurologia, v. 37, n. 7, p. 623-626, 2003.

27. CLIFFORD, T.; FINNERTY, J. The dental awareness and needs of a Parkinson's disease population. Gerodontology, v. 12, n. 2, p. 99-103, 1995.

28. RIBEIRO, Giselle Rodrigues et al. Saúde bucal e função mastigatória em pacientes com doença de Parkinson. 2016. Tese (Doutorado em Clínica Odontológica na área de Prótese Dental) - Faculdade de Odontologia de Piracicaba, Universidade Estadual de Campinas.

29. LOREFÄLT, B. et al. Hábitos alimentares e ingestão de nutrientes em pacientes idosos com doença de Parkinson. Gerontology, v. 52, n. 3, p. 160-168, 2006.

30. VAN STIPHOUT, Marjolein AE et al. Oral Health of Parkinson's Disease Patients: A CaseControl Study. Parkinson's Disease, v. 2018, 2018.

31. DORSEY, ERlet al. Projected number of people with Parkinson disease in the most populous nations, 2005 through 2030. Neurology, v. 68, n. 5, p. 384-386, 2007.

32. YAMAGUCHI, Bruna; FERREIRA, Manoela de Paula; ISRAEL, Vera Lúcia. A multidisciplinaridade na redução da levodopa na pessoa com doença de Parkinson avançada. Acta Fisiatr., v. 23, n.4, p. 197-200, 2016.

33. CICCIU, Marco et al. Periodontal health and caries prevalence evaluation in patients affected by Parkinson's disease. Parkinson's disease, v. 2012, 2012. 